\title{
Cyclically consecutive permutation avoidance
}

\author{
Richard Ehrenborg
}

\begin{abstract}
We give an explicit formula for the number of permutations avoiding cyclically a consecutive pattern in terms of the spectrum of the associated operator of the consecutive pattern. As an example, the number of cyclically consecutive 123-avoiding permutations in $\mathfrak{S}_{n}$ is given by $n$ ! times the convergent series $\sum_{k=-\infty}^{\infty}\left(\frac{\sqrt{3}}{2 \pi(k+1 / 3)}\right)^{n}$ for $n \geq 2$.
\end{abstract}

Key words: Cyclic consecutive pattern avoidance, Integral operators, Spectrum, Trace class operators AMS subject classifications: primary 05A05, secondary 05A15, 45C05

\section{Introduction}

The Euler number $E_{n}$ enumerates the number of alternating permutations in the symmetric group $\mathfrak{S}_{n}$. The Euler number has the following classical expression

$$
E_{n}=n ! \cdot 2 \cdot\left(\frac{2}{\pi}\right)^{n+1} \cdot \sum_{k=-\infty}^{\infty} \frac{1}{(4 k+1)^{n+1}} .
$$

Simons and Yao [8] were the first to the author's knowledge to give a probabilistic-geometric argument for this identity. See also [5, Section 4] and [7]. The underlying idea is that to obtain a random permutation $\pi$ where each permutation is equally likely, pick a random point $x=\left(x_{1}, x_{2}, \ldots, x_{n}\right)$ from the cube $[0,1]^{n}$. Next let the permutation $\pi$ be the standardization of the vector $x$, that is, $\pi_{i}<\pi_{j}$ is equivalent to $x_{i}<x_{j}$ for all indexes $i \neq j$. Geometrically, this corresponds to pick the permutation associated with the simplex containing the point $x$ in the standard triangulation of the cube $[0,1]^{n}$. Hence the probability that the point is alternating, that is, $\cdots<x_{n-2}>x_{n-1}<x_{n}$ is given by $E_{n} / n$ !. By considering the distribution of the last coordinate $f_{n}$ of the point $x$ one obtains the recursion $f_{n}=T\left(f_{n-1}\right)$ where $T$ is the operator given by $T(f)=\int_{0}^{x} f(1-t) d t$. This recursion is straightforward to solve using Fourier series. Integrating this series yields equation (1.1).

Ehrenborg, Kitaev and Perry [4] continued this work by studying consecutive pattern avoidance using operators on the space $L^{2}\left([0,1]^{m}\right)$. The largest eigenvalue of the associated operator yields the asymptotic behavior for the number of permutations. In fact, more knowledge of the eigenvalues gives a better asymptotic expansion; see [4, Theorem 1.1]. However, a complete description of the eigenvalues is hard to find. It is is only known in three cases. First, consecutive 123, 321avoiding permutations, this is, the Euler numbers described above (modulo a factor of 2). Second, consecutive 123-avoiding permutations; see Section 5 of [4]. Finally, in [3, Section 6] a weighted enumeration problem is introduced where the associated operator only has the eigenvalue 1.

In Elkies' argument [7] for the series of the Euler number, he notes that the associated operator is trace class and connects this with cyclically alternating permutations. We show that in the more 
general setting of consecutive pattern avoidance if the operator has powers that are trace class, there is an exact series enumerating the cyclically avoiding permutations. We explicitly state the result for the number of cyclically consecutive 123-avoiding permutations; see Theorem 3.3. Similarly for the weighted enumeration problem, we obtain a closed form formula of $n$ !; see Theorem 3.4 .

We end with open questions for further research.

\section{Weighted enumeration}

For a vector $x=\left(x_{1}, \ldots, x_{k}\right)$ of $k$ distinct real numbers, define $\Pi(x)$ to be standardization of the vector $x$, that is, the unique permutation $\sigma=\left(\sigma_{1}, \ldots, \sigma_{k}\right)$ in $\mathfrak{S}_{k}$ such that for all indices $1 \leq i<j \leq$ $k$ the inequality $x_{i}<x_{j}$ is equivalent to $\sigma_{i}<\sigma_{j}$. Let $S$ be a set of permutations in the symmetric group $\mathfrak{S}_{m+1}$. We say that a permutation $\pi$ in $\mathfrak{S}_{n}$ avoids the set $S$ consecutively if there is no index $1 \leq j \leq n-m$ such that $\Pi\left(\pi_{j}, \pi_{j+1}, \ldots, \pi_{j+m}\right) \in S$. Similarly, a permutation $\pi \in \mathfrak{S}_{n}$ avoids the set $S$ cyclically consecutively if there is no index $1 \leq j \leq n$ such that $\Pi\left(\pi_{j}, \pi_{j+1}, \ldots, \pi_{j+m}\right) \in S$, where the indexes are modulo $n$.

We consider the more general situation of weighted enumeration of consecutive patterns; see [3]. Let wt be a real-valued weight function on the symmetric group $\mathfrak{S}_{m+1}$. Similarly, let $\mathrm{wt}_{1}, \mathrm{wt}_{2}$ be two real-valued weight functions on the symmetric group $\mathfrak{S}_{m}$. We call $\mathrm{wt}_{1}$ and $\mathrm{wt}_{2}$ the initial and final weight function, respectively. We extend these three weight functions to the symmetric group $\mathfrak{S}_{n}$ for $n \geq m$ by defining

$$
\begin{aligned}
\mathrm{Wt}(\pi)= & \mathrm{wt}_{1}\left(\Pi\left(\pi_{1}, \pi_{2}, \ldots, \pi_{m}\right)\right) \\
& \cdot \prod_{i=1}^{n-m} \operatorname{wt}\left(\Pi\left(\pi_{i}, \pi_{i+1}, \ldots, \pi_{i+m}\right)\right) \\
& \cdot \mathrm{wt}_{2}\left(\Pi\left(\pi_{n-m+1}, \pi_{n-m+2}, \ldots, \pi_{n}\right)\right) .
\end{aligned}
$$

Let $\alpha_{n}$ be the sum of all the weights of permutations in $\mathfrak{S}_{n}$, that is,

$$
\alpha_{n}=\sum_{\pi \in \mathfrak{S}_{n}} \mathrm{Wt}(\pi)
$$

This framework can be used to study consecutive pattern avoidance by defining the weight

$$
\operatorname{wt}(\sigma)= \begin{cases}1 & \text { if } \sigma \notin S, \\ 0 & \text { if } \sigma \in S .\end{cases}
$$

Furthermore, let both the initial and final weight functions be the constant function 1. Then for $n \geq m, \alpha_{n}$ is the number of permutations in $\mathfrak{S}_{n}$ that avoid the set $S$.

Define the function $\chi$ on the $(m+1)$-dimensional cube $[0,1]^{m+1}$ by $\chi\left(x_{1}, x_{2}, \ldots, x_{m+1}\right)=$ $\operatorname{wt}\left(\Pi\left(x_{1}, x_{2}, \ldots, x_{m+1}\right)\right)$. The associated linear operator $T$ on the space $L^{2}\left([0,1]^{m}\right)$ is defined by

$$
T(f)\left(x_{1}, \ldots, x_{m}\right)=\int_{0}^{1} \chi\left(t, x_{1}, \ldots, x_{m}\right) \cdot f\left(t, x_{1}, \ldots, x_{m-1}\right) d t .
$$

Similar to $\chi$ define the two functions $\kappa$ and $\mu$ on the $m$-dimensional unit cube $[0,1]^{m}$ by $\kappa(x)=$ $\mathrm{wt}_{1}(\Pi(x))$ and $\mu(x)=\mathrm{wt}_{2}(\Pi(x))$. Then the quantity $\alpha_{n}$ is given by the inner product

$$
\left(T^{n-m}(\kappa), \mu\right)=\alpha_{n} / n !
$$


Hence to study the asymptotic expansion of $\alpha_{n}$ one has to obtain the spectrum of the operator $T$.

We now turn our attention to the power $T^{n}$ where $n \geq m$. Begin by expanding $\chi$ by the product

$$
\chi_{n}\left(x_{1}, x_{2}, \ldots, x_{n}\right)=\prod_{i=1}^{n-m} \chi\left(x_{i}, x_{i+1}, \ldots, x_{i+m}\right) .
$$

Define the kernal $K_{n}(x, y)$ for $x, y \in[0,1]^{m}$ for $x=\left(x_{1}, \ldots, x_{m}\right)$ and $y=\left(y_{1}, \ldots, y_{m}\right)$ by the integral

$$
K_{n}(x, y)=\int_{[0,1]^{n-m}} \chi_{n+m}\left(x_{1}, \ldots, x_{m}, t_{1}, \ldots, t_{n-m}, y_{1}, \ldots, y_{m}\right) d t_{1} \cdots d t_{n-m}
$$

Since

$$
T^{n} f(y)=\int_{[0,1]^{m}} K_{n}(x, y) d x .
$$

we conclude that $T^{n}$ is a Hilbert-Schmidt operator, and hence $T^{n}$ is compact.

\section{$3 \quad$ Weighted cyclic enumeration}

Define the cyclic weight of a permutation $\pi$ in $\mathfrak{S}_{n}$, where $n \geq m+1$, by the product

$$
\mathrm{Wt}^{c}(\pi)=\prod_{i=1}^{n} \mathrm{wt}\left(\Pi\left(\pi_{i}, \pi_{i+1}, \ldots, \pi_{i+m}\right)\right),
$$

where the indexes are modulo $n$. Similar to $\alpha_{n}$, we consider the sum of cyclic weights to be

$$
\beta_{n}=\sum_{\pi \in \mathfrak{S}_{n}} \mathrm{Wt}^{c}(\pi)
$$

We will write $\beta_{n}(S)$ when the weighting function is associated with avoiding a certain pattern $S$.

Lemma 3.1. The sum of the cyclic weights $\beta_{n}$ is given by the integral

$$
\beta_{n}=n ! \cdot \int_{[0,1]^{n}} \chi_{n+m}\left(x_{1}, x_{2}, \ldots, x_{n}, x_{1}, \ldots, x_{m}\right) d x_{1} \cdots d x_{n} .
$$

Proof. We evaluate the integral by partitioning the $n$-dimensional cube by the standard triangulation and noting that the function $\chi_{n+m}$ is constant on each of the $n$ ! simplexes, that is,

$$
\begin{aligned}
& \int_{[0,1]^{n}} \chi_{n+m}\left(x_{1}, x_{2}, \ldots, x_{n}, x_{1}, \ldots, x_{m}\right) d x_{1} \cdots d x_{n} \\
& =\sum_{\pi \in \mathfrak{S}_{n}} \int_{\substack{x \in[0,1]^{n} \\
\Pi(x)=\pi}} \chi_{n+m}\left(x_{1}, x_{2}, \ldots, x_{n}, x_{1}, \ldots, x_{m}\right) d x_{1} \cdots d x_{n} \\
& =\sum_{\pi \in \mathfrak{S}_{n}} \int_{\substack{x \in[0,1]^{n} \\
\Pi(x)=\pi}} \mathrm{Wt}^{c}(\pi) d x_{1} \cdots d x_{n} \\
& =\frac{1}{n !} \cdot \sum_{\pi \in \mathfrak{S}_{n}} \mathrm{Wt}^{c}(\pi) .
\end{aligned}
$$


Theorem 3.2. Let $\left(\lambda_{k}\right)_{1 \leq k \leq K}$, where $K \leq \infty$, be a list of the eigenvalues of the operator $T$ (including multiplicities), where $T$ has the form given in equation (2.11). Let $n \geq m+1$. Assume that the series $\sum_{k} \lambda_{k}^{n}$ converges absolutely. Then the sum of the cyclic weights is given by

$$
\beta_{n}=n ! \cdot \sum_{k=1}^{K} \lambda_{k}^{n}
$$

Proof. Begin to observe that $T^{n}$ is an Hilbert-Schmidt operator. Since the sum $\sum_{k}\left|\lambda_{k}\right|^{n}$ converges then the operator $T^{n}$ is a trace class operator; see Section XI.8, Exercise 49 in [1]. The trace of the operator is given by the convergent sum

$$
\operatorname{tr}\left(T^{n}\right)=\sum_{k=1}^{K} \lambda_{k}^{n}
$$

But the trace of $T^{n}$ is also given by the following integral; see part (c) of the above mentioned exercise in [1]:

$$
\begin{aligned}
\operatorname{tr}\left(T^{n}\right) & =\int_{[0,1]^{m}} K(x, x) d x \\
& =\int_{[0,1]^{n}} \chi_{n+m}\left(x_{1}, \ldots, x_{m}, t_{1}, \ldots, t_{n-m}, x_{1}, \ldots, x_{m}\right) d x_{1} \cdots d x_{m} d t_{1} d t_{n-m} \\
& =\beta_{n} / n !
\end{aligned}
$$

proving the result.

We give two examples.

Theorem 3.3. The number of cyclically consecutive 123-avoiding permutations in the symmetric group $\mathfrak{S}_{n}$ for $n \geq 2$ is given by

$$
\beta_{n}(123)=n ! \cdot \sum_{k=-\infty}^{\infty}\left(\frac{\sqrt{3}}{2 \pi(k+1 / 3)}\right)^{n} .
$$

Proof. Here we let the weight function wt on $\mathfrak{S}_{3}$ be given by wt $(\sigma)=1-\delta_{\sigma, 123}$, where $\delta$ denotes the Kronecker delta. The eigenvalues are given by $\lambda_{k}=\frac{\sqrt{3}}{2 \pi(k+1 / 3)}$ where $k$ ranges over all the integers; see [4, Proposition 5.1]. Note for $n \geq 2$ the series $\sum_{k=-\infty}^{\infty}\left|\lambda_{k}\right|^{n}$ converges. By Theorem 3.2 the result follows in the case $n \geq 3$. The case $n=2$ is can be verified by a separate calculation.

A different approach to prove this theorem is to cyclically shift the permutations such that $\pi_{n}=n$. Now we are looking for 123-avoiding permutations $\pi_{1} \cdots \pi_{n-1}$ in $\mathfrak{S}_{n-1}$. However, we also require that the last two entries yields a descent, since otherwise the three entries $\pi_{n-2} \pi_{n-1} \pi_{n}$ would have the 123 pattern. The number of such permutations in $\mathfrak{S}_{n-1}$ can be calculated by $(n-1) ! \cdot\left(T^{n-m}(\kappa), \mu\right)$ where $\kappa$ is the constant function 1 and $\mu$ is the 0,1 -function $\mu(x, y)=1$ if $x>y$ and $\mu(x, y)=0$ if $x<y$. However, Theorem 2.1 in [3] only gives an asymptotic expansion 
for the desired quantity. Lastly, one has to do an analytic argument to show that the asymptotic expansion actually gives a convergent series.

As our next example, we consider the weighted example from [3, Section 6]. Let $\mathbf{b b}(\pi)$ and $\mathbf{b b}^{c}(\pi)$ denote the number of double descents of the permutation $\pi$, respectively, the number of cyclically double descents of the permutation $\pi$, that is,

$$
\begin{aligned}
\mathbf{b b}(\pi) & =\#\left\{i \in\{1,2, \ldots, n-2\}: \pi_{i}<\pi_{i+1}<\pi_{i+2}\right\}, \\
\mathbf{b b}^{c}(\pi) & =\#\left\{i \in\{1,2, \ldots, n\}: \pi_{i}<\pi_{i+1}<\pi_{i+2}\right\},
\end{aligned}
$$

where the indexes are modulo $n$.

Theorem 3.4. The sum of $2^{\mathbf{b b}^{c}(\pi)}$ over all permutations $\pi$ in $\mathfrak{S}_{n}$ which cyclically do not have a double ascent is given by $n$ !, that is,

$$
\sum_{\substack{\pi \in \mathfrak{S}_{n} \\ \pi \text { cyclically 123-avoiding }}} 2^{\mathbf{b b}^{c}(\pi)}=n ! .
$$

Proof. Here the weight function wt on $\mathfrak{S}_{3}$ be given by $\operatorname{wt}(132)=\operatorname{wt}(213)=\operatorname{wt}(231)=\operatorname{wt}(312)=1$, $\operatorname{wt}(123)=0$ but $\operatorname{wt}(321)=2$. The associated operator has only one eigenvalue namely 1 ; see [3, Theorem 6.1]. Hence the result follows directly for $n \geq 3$. The three cases $n \leq 2$ are direct.

Corollary 3.5. The sum of $2^{\mathbf{b b}(\pi)}$ over all permutations in $\mathfrak{S}_{n}$ such that $\pi_{1}=n$, there is no double ascent and which end with a descent, that is, $\pi_{n-1}>\pi_{n}$, is given by $(n-1)$ !.

Proof. The weight of a permutation in the previous theorem does not change when it is cyclically shifted. Hence shift the permutation such that $\pi_{1}=n$ and the sum is given by $n ! / n$.

As an example, there are 9 permutations in $\mathfrak{S}_{5}$ that are 123-avoiding, begin with the element 5 and end with a descent. In lexicographic order they are 51432, 52143, 52431, 53142, 53241, 53421, 54132,54231 , and 54321. Note that the 8 first permutations all have one double descent and the last permutation has three double descents yielding the sum $8 \cdot 2^{1}+1 \cdot 2^{3}=4$ !.

It is tempting to use [4, Proposition 7.2] to obtain a result for the number of cyclically consecutive 123,231,312-avoiding permutations. However, note that this proposition only determines a part of the spectrum of the associated operator corresponding to an invariant subspace. Hence there is not enough information to apply Theorem 3.2 .

\section{Concluding remarks}

Are there other operators of the form (2.1) where we can determine the spectrum? This is not a straightforward question since the spectrum for 213-avoiding permutations is given by the equation

$$
\operatorname{erf}\left(\frac{1}{\sqrt{2} \cdot \lambda}\right)=\sqrt{\frac{2}{\pi}},
$$

where erf denotes the error function. Is is always true that the sum $\sum_{k}\left|\lambda_{k}\right|^{m}$ is absolutely convergent? In other words, is the operator $T^{m}$ always trace class? Does the generating function $\sum_{n \geq 0} \beta_{n} \cdot z^{n} / n$ ! have a nicer form than the generating function $\sum_{n \geq 0} \alpha_{n} \cdot z^{n} / n$ !; see [2, 6]. 
It is interesting to note that Theorem 3.3 gives a exact expression for the number of cyclically consecutive 123-avoiding permutations. However, for consecutive 123-avoiding permutations the corresponding result [4, Theorem 5.4] only yields an asymptotic expansion.

Since this result of Theorem 3.4 is purely combinatorial, it is natural to ask for a bijective proof.

\section{Acknowledgments}

The author is grateful to Margaret Readdy for her comments on an earlier version of this paper. The author was partially supported by National Security Agency grant H98230-13-1-0280.

\section{References}

[1] N. Dunford and J. Schwartz, "Linear operators. Part II. Spectral theory. Selfadjoint operators in Hilbert space. With the assistance of William G. Bade and Robert G. Bartle. Reprint of the 1963 original. Wiley Classics Library.," A Wiley-Interscience Publication. John Wiley \& Sons, Inc., New York, 1988.

[2] R. EhrenborG, On the generating function for consecutively weighted permutations, preprint 2012 .

[3] R. Ehrenborg And J. Jung, Descent pattern avoidance, Adv. in Appl. Math. 49 (2012), $375-390$.

[4] R. Ehrenborg, S. Kitaev And P. Perry, A spectral approach to consecutive patternavoiding permutations, J. Comb. 2 (2011), 305-353.

[5] R. Ehrenborg, M. Levin and M. Readdy, A probabilistic approach to the descent statistic, J. Combin. Theory Ser. A 98 (2002), 150-162.

[6] S. Elizalde And M. Noy, Clusters, generating functions and asymptotics for consecutive patterns in permutations, Adv. in Appl. Math 49 (2012), 351-374.

[7] N. D. Elkies, On the sums $\sum_{k=-\infty}^{\infty}(4 k+1)^{-n}$, Amer. Math. Monthly 110 (2003), 561-573.

[8] G. Simons And Y.-C. YaO, Asymptotic sine laws arising from alternating random permutations and sequences, Random Structures Algorithms 8 (1996), 75-95.

R. Ehrenborg, Department of Mathematics, University of Kentucky, Lexington, KY 40506-0027, jrge@ms.uky.edu 